\title{
Computation of Surface Catalysis for Graphite Exposed to High-Enthalpy Nitrogen Flow
}

\author{
Abhilasha Anna* and Iain D. Boyd ${ }^{\dagger}$ \\ Department of Aerospace Engineering, University of Michigan, Ann Arbor, MI, 48109
}

\begin{abstract}
The high temperatures on a hypersonic vehicle surface caused by heat loads encountered during (re-)entry through a planetary atmosphere require a reliable Thermal Protection System (TPS) that makes a good understanding of the physical and chemical processes essential for its design. Surface catalysis is a crucial chemical process that directly impacts aerothermal heating of the vehicle TPS. To study the effects of this process, a binary catalytic atom recombination model is implemented in a computational fluid dynamics (CFD) code. The study examines the effects of surface catalysis for graphite exposed to high enthalpy nitrogen flow. As expected, surface catalysis strongly affects the boundary layer gradients of temperature and species concentration, and heat transfer to the surface. A fully catalytic surface causes the heat flux to increase by a factor of approximately 3.5. The physical accuracy of the model is assessed using data from experimental tests conducted in the Inductively Coupled Plasma (ICP) Torch Facility at the University of Vermont. Comparisons are presented of computed results with measured experimental data for translational temperature and relative nitrogen atom number density in the flow in front of the test article.
\end{abstract}

\section{Nomenclature}

$D_{12} \quad$ binary diffusion coefficient between species 1 and $2\left[\mathrm{~m}^{2} / \mathrm{s}\right]$

$J_{s p} \quad$ species diffusion flux $\left[\mathrm{kg} / \mathrm{m}^{2} / \mathrm{s}\right]$

$\dot{M} \quad$ mass flux $\left[\mathrm{kg} / \mathrm{m}^{2} / \mathrm{s}\right]$

$N_{s p} \quad$ number of species in the mixture [dimensionless number]

$T \quad$ translational-rotational temperature $[\mathrm{K}]$

$Y \quad$ mass fraction [dimensionless number]

$h_{s p} \quad$ species enthalpy $[\mathrm{J} / \mathrm{kg}]$

$k_{B} \quad$ Boltzmann constant

$k_{w} \quad$ wall catalytic speed $[\mathrm{m} / \mathrm{s}]$

$m$ particle mass [kg]

$q \quad$ total heat flux $\left[\mathrm{W} / \mathrm{m}^{2}\right]$

$q_{\text {conv }} \quad$ convective heat flux $\left[\mathrm{W} / \mathrm{m}^{2}\right]$

$q_{\text {diff }} \quad$ diffusive heat flux $\left[\mathrm{W} / \mathrm{m}^{2}\right]$

$\gamma \quad$ wall catalytic efficiency [dimensionless number]

$\kappa \quad$ thermal conductivity $[\mathrm{W} / \mathrm{m} / \mathrm{K}]$

$\rho \quad$ density $\left[\mathrm{kg} / \mathrm{m}^{3}\right]$

subscripts

$w \quad$ wall value

tr translational-rotational energy mode

ve vibrational-electronic energy mode

$\infty \quad$ reference freestream conditions

$s p \quad$ species value

${ }^{*}$ Graduate Student, Student Member AIAA.

${ }^{\dagger}$ James E. Knott Professor, Fellow AIAA. 


\section{Introduction}

H YPERSONIC vehicles experience heat loads during high-speed flight through the atmosphere that cause H very high temperatures on their surface. Therefore, such vehicles use a Thermal Protection System (TPS) to protect them from aerodynamic heating and maintain acceptable temperatures on their surface. The prolonged exposure to high temperature and chemical reactions can cause the TPS materials to fail. ${ }^{1}$ The need to design a reliable TPS necessitates good understanding of the physical and chemical processes that determine the aerothermal heating environment.

Depending on the heat load encountered during hypersonic flight, an ablative or non-ablative TPS could be used. ${ }^{2}$ Non-ablative or reusable materials are used where the re-entry conditions are mild while ablative TPS materials are used where high heating rates are generated. There is no change in mass or properties of the non-ablative material whereas ablative TPS materials accommodate high heating rates and heat loads through phase change and mass loss. Ablative TPS has been used for most planetary entry probes and high velocity Earth atmosphere re-entry vehicles. An ablative TPS surface can be heated ${ }^{3}$ by (1) convective heating as a result of gas particle collisions and their interactions with the surface and (2) radiative heating as a result of radiation from excited particles in the flow. Catalycity of an ablative TPS material is one of the factors that impacts the heating of the vehicle surface. Very high temperatures in the boundary layer can cause the gas species to dissociate. If the heated TPS material acts as a catalyst and dissociated atoms diffuse to the surface, it can cause recombination of dissociated boundary layer species which increases the convective heating to the surface. Thus, a less catalytic surface is desirable to minimize this additional heating. Also, when the vehicle surface is heated, the surface material may chemically react with the boundary layer gases and can lead to surface recession as a result of surface material consumption. These chemical reactions can be endothermic (vaporization, sublimation) or exothermic (oxidation) and will affect the net heating to the surface. Therefore, detailed studies of these interactions that occur between the surface and the atmosphere gas are required.

Accurate modeling of surface catalysis is a key factor in the prediction of aerothermal heating of the vehicle TPS and in characterizing TPS materials. The accurate prediction of the aerothermal environment is necessary to determine the TPS sizing, uncertainties in which could lead to an over-sized TPS. ${ }^{4}$ The objective of this research is to implement and assess a surface catalysis model for investigating the effect of catalycity on the species concentration and temperature gradients near the material surface, and on the heat transfer to the material surface.

To study the effects of surface catalysis, the entry flight environment considered is the post shock subsonic high enthalpy gas flow. To characterize a TPS material, it needs to be tested in an experimental facility that can create conditions representative of real entry flight conditions. It is challenging and expensive to fully reproduce real flight conditions in existing ground facilities. ${ }^{5}$ Therefore, a partial simulation of the flight conditions that involves subsonic high enthalpy flow for the purpose of this study is considered. This paper describes the numerical approach used to simulate these conditions along with the experimental approach taken to assess the computational results. Figure 1 shows a full hypersonic flowfield computed around a capsule taken from Ref. 6 . The insert shows the post-shock, high-enthalpy, subsonic region considered in the present study. The comparative analysis of the computed profiles of gas temperature and relative nitrogen atom density with the experimental tests conducted at the University of Vermont is presented. The paper ends with some conclusions and suggestions for future work.

\section{Technical Approach}

\section{A. Numerical Method}

The numerical simulations in this study are conducted using the Michigan Aerothermodynamic NavierStokes computational fluid dynamics (CFD) code LeMANS ${ }^{7-9}$ developed at the University of Michigan. It is a general purpose, parallel, three-dimensional code that solves the laminar Navier Stokes equations including chemical and thermal nonequilibrium effects on unstructured computational grids. The flow is modeled assuming the continuum approximation is valid. The set of partial differential equations are solved using a finite-volume method on unstructured grids. The inviscid fluxes across cell faces are discretized using a modified form of the Steger-Warming Flux Vector Splitting (FVS) scheme ${ }^{10}$ which is less dissipative and produces better results in boundary layers. The viscous terms are calculated using the values of properties at the cell centers and at the nodes. Time integration is performed using a point implicit or a line implicit 


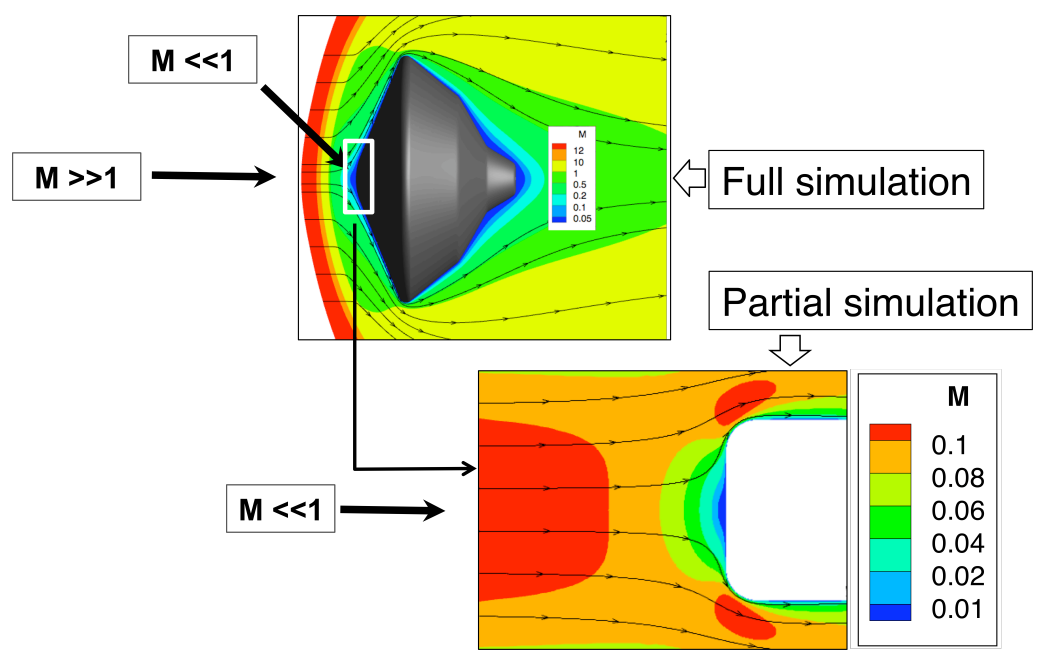

Figure 1. Flow regime investigated

method. LeMANS is parallelized using METIS $^{11}$ that partitions the computational grid between the processors and Message Passage Interface protocol (MPI) to communicate information between processors. It is assumed that the translational and rotational energy modes of all species can be described by two different temperatures $\mathrm{T}$ and $\mathrm{T}_{r}$ respectively, while the vibrational energy mode and electron energy of all species can be described by a single temperature $\mathrm{T}_{v} \cdot{ }^{12}$ LeMANS can simulate two-dimensional/axisymmetric flows using any mixture of quadrilaterals and triangles, and three-dimensional flows using any mixture of hexahedra, tetrahedral, prisms and pyramids. All the simulations are performed in this study using computational grids that contained quadrilateral elements.

\section{Species boundary conditions}

Surface catalycity effects can be accounted for by four types of conditions at the wall boundary as shown in Table 1. In LeMANS, prior to the present work, wall catalycity effects were accounted for by choosing a non-catalytic or a super-catalytic surface as the species boundary condition. A super-catalytic boundary condition is inappropriate for the present flows that are comprised of dissociated species as this condition will lead to the same composition at the wall as in the free stream which is undesirable for conditions where the effect of recombination on the heat transfer is to be studied. For the simulation of the full range of catalycity

Table 1. Species boundary conditions.

\begin{tabular}{|l|l|}
\hline Boundary Condition & Description \\
\hline \hline Non-catalytic & No recombination of atoms on the surface \\
\hline Super-catalytic & $\begin{array}{l}\text { Atoms that strike the surface recombine to the } \\
\text { free stream gas composition }\end{array}$ \\
\hline Fully-catalytic & $\begin{array}{l}\text { All atoms that strike the surface recombine to } \\
\text { form molecules }\end{array}$ \\
\hline Partially-catalytic & Some atoms reflect and some recombine \\
\hline
\end{tabular}

regimes, from a non-catalytic wall to a fully-catalytic wall, a simplified catalytic atom recombination model, i.e. a binary interaction model with full energy accommodation, ${ }^{13,14}$ is implemented in LeMANS. It is implemented by balancing the mass flux of the relevant species taking the consumption/production at the wall into account. The model is applied to a species boundary condition by considering a first order recombination reaction for a binary gas at the wall. The boundary condition for the mass fraction $\mathrm{Y}_{N}$ of atomic nitrogen 
and $\mathrm{Y}_{N_{2}}$ of molecular nitrogen in a binary mixture $\left(\mathrm{N}_{2}-\mathrm{N}\right)$ is given by the expression shown in Eq. 1.

$$
\begin{aligned}
D_{12} \frac{\partial Y_{N}}{\partial n} & =k_{w N} Y_{N} \\
Y_{N_{2}} & =1-Y_{N}
\end{aligned}
$$

where $D_{12}$ is the binary diffusion coefficient between atomic and molecular species, $n$ is the wall normal direction and $k_{w_{N}}$ is the catalytic recombination speed given by the Hertz-Knudsen relation shown in Eq. 2 .

$$
k_{w N}=\gamma_{N} \sqrt{\frac{k_{B} T}{2 \pi m_{N}}}
$$

In this model, the atoms that impinge on a surface either reflect from the surface and neither gain nor lose energy at the surface, or become adsorbed where an atom may react with another atom to produce a molecule. In the latter case, the energy released by the reaction, which is highly exothermic, is assumed to be entirely transferred to the wall and this phenomenon is called full accommodation. In this model, a dimensionless surface recombination coefficient ${ }^{13,15,16} \gamma$ (also referred to as the catalytic efficiency) is introduced. It is defined as the ratio of the flux of atoms that recombine on the surface $\dot{M}_{\text {rec }}$ to the total flux of atoms that impinge on the surface $\dot{M}_{i m p}$ and the expression is shown in Eq.3.

$$
\gamma=\frac{\dot{M}_{r e c}}{\dot{M}_{i m p}}
$$

This coefficient depends on the particular atom and surface involved. If the wall is fully-catalytic, then $\gamma_{N}$ and the corresponding boundary condition for the atom becomes,

$$
\begin{gathered}
\gamma_{N}=1 \\
Y_{N}=0
\end{gathered}
$$

If the wall is non-catalytic, then $\gamma_{N}$ is set to zero that implies the species concentration gradient is zero at the wall (Eqn. 5). If the wall is partially catalytic, $\gamma_{N}$ is set to a value between 0 and 1 based on the catalycity of the surface.

$$
\frac{\partial Y_{N}}{\partial n}=-\frac{\partial Y_{N_{2}}}{\partial n}=0
$$

\section{Thermochemical model}

For this study, nitrogen gas mixture is considered composed of atomic and molecular nitrogen using the following dissociation-recombination reaction:

$$
\begin{aligned}
& N_{2}+M \rightleftharpoons 2 N+M \\
& M=N, N_{2}
\end{aligned}
$$

The mixture transport properties are modeled using Wilke's semi-empirical mixing rule ${ }^{17}$ with species viscosities calculated using Blottner's model ${ }^{18}$ and the species thermal conductivities are determined using Eucken's relation. ${ }^{19}$ The species mass production rates are modeled using a finite-rate chemistry model. ${ }^{20}$ In this study, dissociation reactions are modeled using Park's two-temperature model wherein it is assumed that the rotational and translational energy modes of all species can be described by a single temperature $\mathrm{T}_{t r}$ and the vibrational energy mode of all species and the electron energy can be described by a single temperature $\mathrm{T}_{v e}{ }^{21}$

\section{Heat flux at the wall}


species to the surface. The convective heat flux is composed of convection $\mathrm{q}_{\text {conv,tr }}$ due to the translational and rotational modes and $\mathrm{q}_{c o n v, v e}$ due to the vibrational mode when thermal nonequilibrium is accounted 
for. The convective heat flux is modeled according to Fourier's law and species diffusion heat fluxes are modeled using a modified form of Fick's law. ${ }^{22}$ They are given by the following expressions:

$$
\begin{aligned}
& q=q_{\text {conv }}+q_{d i f f} \\
& q_{\text {conv }, t r, v e}=-\kappa_{t r, v e} \frac{\partial T_{t r, v e}}{\partial n} \\
& q_{\text {diff }}=\sum_{i=1}^{N_{s p}} h_{i} J_{i}
\end{aligned}
$$

where $\kappa_{t r, v e}$ is the thermal conductivity for each energy mode, $\mathrm{h}_{i}$ is the species enthalpy and $\mathrm{J}_{i}$ is the normal species diffusion flux. The wall is assumed isothermal and is set to the prescribed wall temperature $\mathrm{T}_{w}$.

\section{B. Experimental Technique}

Assessment of the computations is performed using experimental tests that were conducted in the $30 \mathrm{~kW}$ Inductively Coupled Plasma (ICP) Torch Facility ${ }^{23,24}$ at the University of Vermont. It is designed to test scaled material samples in high enthalpy gas flows for simulation of planetary entry and Earth atmosphere re-entry trajectory heating conditions. It is configured for operation with subsonic flow to simulate post shock conditions of high enthalpy flight for a stagnation point geometry. An advantage of the inductively coupled approach is that the free stream is usually free of metal contaminants (e.g. copper) as this approach is electrode-less as opposed to using arc jets in which particles from the electrodes are often found in the free stream. ${ }^{1}$

The primary components of the facility are the power supply, plasma generation system and the test chamber. The power is supplied by an induction heating generator that can deliver up to $30 \mathrm{~kW}$ of energy. The plasma generation system is comprised of the inductance coil, quartz confinement tube and gas injection system. A radio frequency electrical power is supplied to the 6 turn helical coil that provides an oscillating magnetic field within the $36 \mathrm{~mm}$ internal diameter quartz confinement tube. A gas injection system delivers test gas that enters the confinement tube and thus hot plasma gas is generated. This hot plasma flows out of the quartz tube into the test chamber towards the mounted test article. The design and the fabrication of the facility is described in detail in the work by Owens et al. ${ }^{24}$

For this study, experimental results from POCO graphite grade DFP2 samples tested in the nitrogen plasma stream are used. Most ablative TPS materials are organic composites that makes it necessary to study interaction with carbon based materials. The experimental tests were aimed at measuring the nitrogen atom number density and translational temperature in the reacting boundary layer above the graphite surface using a two-photon laser induced fluorescence (LIF) technique. A two color infrared optical pyrometer was used to measure surface temperature and sample ablation was also quantified by determining carbon mass loss from pre-and post-test mass measurements.

\section{Numerical Setup}

The test article is a $19.05 \mathrm{~mm}$ diameter graphite sample mounted at a distance of $116 \mathrm{~mm}$ from the quartz tube exit. Hot nitrogen plasma flows out through the quartz tube at a flow rate of 38.1 standard liters/minute. Figure 2 shows the graphite sample during exposure to the nitrogen plasma in the ICP Torch Facility. The free stream conditions and wall temperature used for this study are based on the experimental set up and are provided in Table 2. The free stream Knudsen number for these conditions is calculated to be $5.6 \times 10^{-4}$ indicating that the flow is in the continuum regime. The diameter of the test sample is chosen to be the characteristic length. The free stream Reynolds number for this flow is 512 indicating that the flow is laminar. The characteristic length chosen is the length of the test article plus the length of the holder.

Table 2. Freestream and wall boundary conditions.

\begin{tabular}{|l|l|l|l|l|l|}
\hline $\begin{array}{l}\text { Mach Number, } \\
M_{\infty}\end{array}$ & $\begin{array}{l}\text { Temperature, } \\
T_{\infty}[\mathrm{K}]\end{array}$ & $\begin{array}{l}\text { Density, } \rho_{\infty} \\
{\left[\mathrm{kg} / \mathrm{m}^{3}\right]}\end{array}$ & $\begin{array}{l}\mathrm{N}_{2} \text { Density, } \rho_{N_{2}} \\
{\left[\mathrm{~kg} / \mathrm{m}^{3}\right]}\end{array}$ & $\begin{array}{l}\mathrm{N} \text { Density, } \rho_{N \infty} \\
{\left[\mathrm{kg} / \mathrm{m}^{3}\right]}\end{array}$ & $\begin{array}{l}\text { Wall Temperature, } \\
T_{w}[\mathrm{~K}]\end{array}$ \\
\hline \hline 0.11 & 7000 & $3.36 \times 10^{-3}$ & $0.35 \times 10^{-3}$ & $3.01 \times 10^{-3}$ & 1590 \\
\hline
\end{tabular}




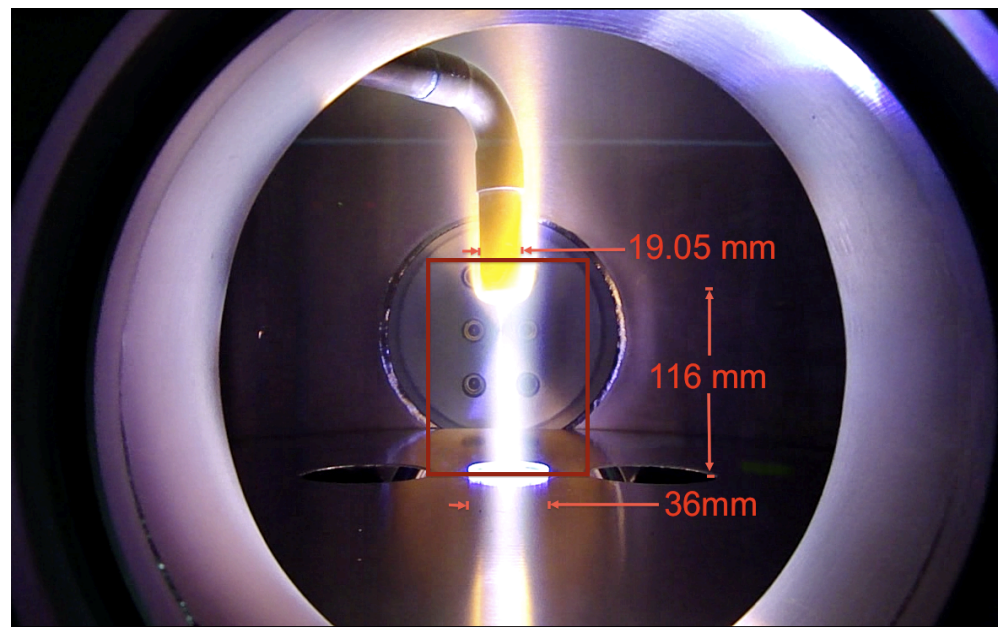

Figure 2. Experimental set up with graphite sample in nitrogen plasma (section in box is the portion simulated) (Source: Prof. D.G. Fletcher ${ }^{25}$ ).

The equilibrium composition of nitrogen gas mixture for the given temperature and pressure ${ }^{23}$ are calculated using the NASA program Chemical Equilibrium with Applications ${ }^{26}$ (CEA). This program can compute the chemical equilibrium compositions of mixtures for assigned thermodynamic states. The states may be specified by assigning two thermodynamic state variables, which are temperature $(7000 \mathrm{~K})$ and pressure $(95$ Torr) for this study. The results of this computation show a significant level of nitrogen dissociation. The prominent species present in the gas mixture are molecular and atomic nitrogen. Other species present are $\mathrm{N}^{+}, \mathrm{N}_{2}+$ and $\mathrm{e}^{-}$which are not included in the computation because of their negligible quantities. The high temperature flow field is examined for thermal and thermochemical nonequilibrium effects. The plasma exit velocity is calculated to be $231 \mathrm{~m} / \mathrm{s}$ from the given volume flow rate of 38 std liters per minute of nitrogen $\left(N_{2}\right)$ gas. The boundary conditions set for the simulation are shown in Fig. 3. The test chamber wall is constructed of stainless steel and is water cooled. It is set as a non-catalytic wall ${ }^{27,28}$ with a wall temperature of $300 \mathrm{~K}$. The enthalpy of the flow is calculated to be $37 \mathrm{MJ} / \mathrm{kg}$ based on CEA values for equilibrium conditions. The catalycity model is applied to the test article surface. The grid is generated using the commercial mesh generation software Pointwise. ${ }^{29}$ Grid independence is achieved for a flow in thermal equilibrium using a grid with 113,000 quadrilateral cells. This grid is used for all the test cases studied.

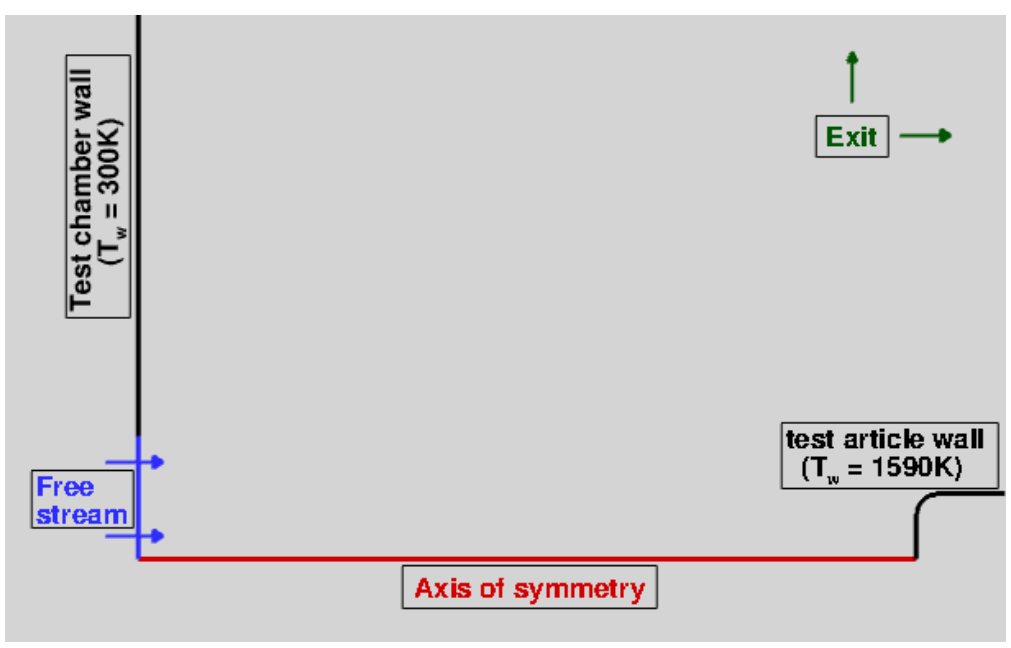

Figure 3. Boundary conditions 


\section{Results}

In this section, the results of the study that use the CFD code LeMANS to determine surface catalycity effects on a graphite sample exposed to a high enthalpy and low pressure flow are provided. The flow physics models and the species catalytic efficiency $\gamma_{N}$ used for each test case are shown in Table 3. The test cases are chosen to study the influence of different flow physics assumptions and surface catalysis on the numerical solution. The three flow physics models are defined as follows. A flow in thermal equilibrium (TE) means that all energy modes have the same temperature and the flow is not chemically reacting. Thermal nonequilibrium (TNE) means that the flow considers vibrational nonequilibrium and the flow is not chemically reacting. In this case, the finite rate of vibrational relaxation is accounted for. The translational and rotational modes are assumed to be equilibrated. Thermochemical nonequilibrium (TCNE) means that the flow is both in vibrational and chemical nonequilibrium. With these assumptions, the finite rate of chemical relaxation is included, along with vibrational relaxation. The catalytic efficiency $\gamma_{N}$ is set to zero for a non-catalytic wall and is set to 1 for a fully-catalytic wall. The main calculated parameters analyzed are translational temperature, relative nitrogen atom density, and surface heat flux.

Table 3. Different flow field conditions used.

\begin{tabular}{|l|l|l|}
\hline Case & Flow field condition & Catalytic efficiency $\gamma_{N}$ \\
\hline \hline Case 1 & Thermal equilibrium (TE) & $\gamma_{N}=0$ \\
\hline Case 2 & Thermal nonequilibrium (TNE) & $\gamma_{N}=0$ \\
\hline Case 3 & Thermochemical nonequilibrium (TCNE) & $\gamma_{N}=0$ \\
\hline Case 4 & Thermal nonequilibrium (TNE) & $\gamma_{N}=1$ \\
\hline Case 5 & Thermochemical nonequilibrium (TCNE) & $\gamma_{N}=1$ \\
\hline
\end{tabular}

The comparisons between the numerical results and experimental LIF measurements are presented for translational temperature and relative nitrogen atom density in the test sample boundary layer along the stagnation streamline and in the radial direction at a distance of $1.52 \mathrm{~mm}$ from the test article surface. The relative nitrogen atom density is calculated by scaling the numerical profile obtained from LeMANS for Case 5 (which is expected to provide the best agreement with the measurements) to agree with the measurement at the largest distance from the test sample and then the scaled factor is used to obtain the relative axial stagnation line and radial profiles for all five simulation conditions. The stagnation line boundary layer results are shown in Fig. 4, with translational temperature in Fig. 4(a) and the relative nitrogen atom density in Fig. 4(b). The radial profile results for translational temperature and relative nitrogen atom number density are shown in Figs. 5(a) and 6(a), respectively. Figure 7 presents the calculated wall heat fluxes for different flow field conditions and values of $\gamma$. The total heat flux is shown in Fig. 7(a) and the components that contribute to the total heat flux, viz. translational convective heat flux, vibrational convective heat flux and diffusive heat flux, are shown in Figs. $7(\mathrm{~b}), 7(\mathrm{c})$ and $7(\mathrm{~d})$, respectively. The results are discussed in three sections. Section A presents the thermal and thermochemical nonequilibrium effects. The effects of a fully-catalytic boundary condition and comparison with the experimental measurements are discussed in Section B. The possible causes of nitrogen atom loss observed in the experiment are discussed in Section C.

\section{A. Effect of thermal and thermochemical nonequilibrium}

Results for the simulations run with different flow field physics models described in Table 3 for a noncatalytic $\left(\gamma_{N}=0\right)$ surface, i.e. Cases 1, 2 and 3, are discussed in this section. Thermal nonequilibrium effects are evaluated for the vibrational mode. The simulation run time for thermal equilibrium, thermal nonequilibrium and thermochemical nonequilibrium cases are approximately 90, 180 and 185 hours with each simulation using 16,32, and 32 processors, respectively. The simulation for Cases 1 and 2 show no significant difference in the results and therefore, results for Case 1, i.e. thermal equilibrium, are not presented. The reason for this agreement between the two cases is that at this high temperature of $7000 \mathrm{~K}$, the flow is mostly dissociated. The fraction of total energy contained in the vibrational mode of the molecules is therefore small, and hence the vibrational nonequilibrium effects are negligible for a non-catalytic wall.

For the case of thermochemical nonequilibrium, a rise in temperature in the boundary layer is seen both in the axial and radial profiles as shown in Figs. 4(a) and 5(a), respectively. The axial and radial relative 


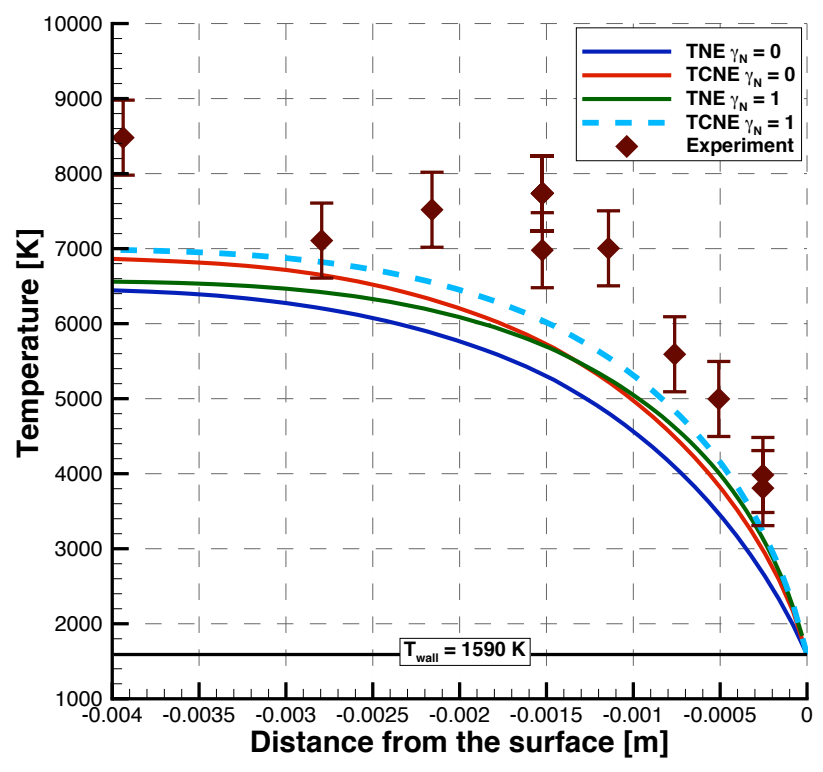

(a) Translational temperature

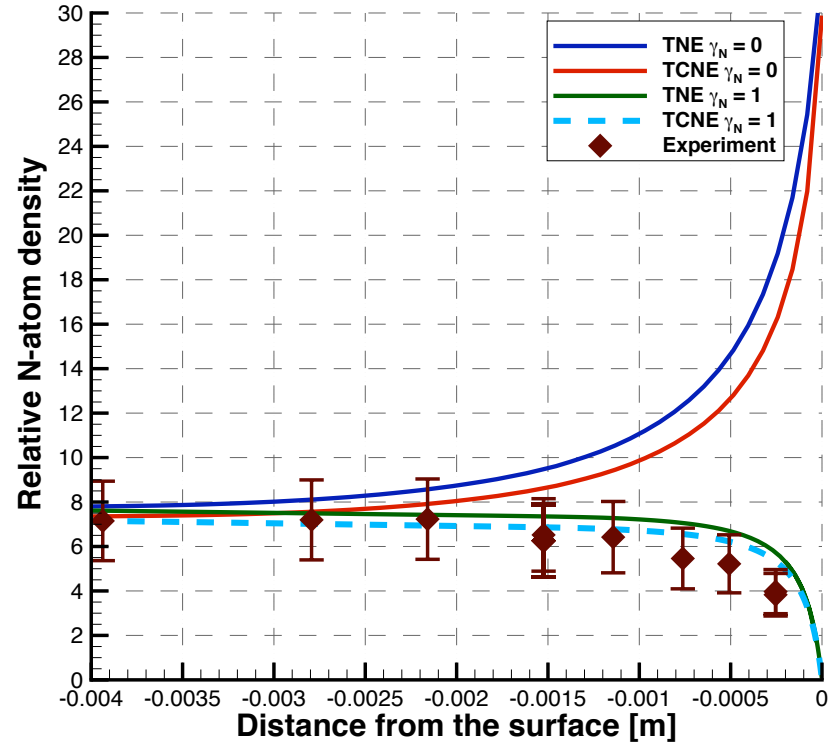

(b) Relative $\mathrm{N}$-atom density

Figure 4. Comparison of translational temperature and relative N-atom density along the stagnation line between the computational and experimental data.

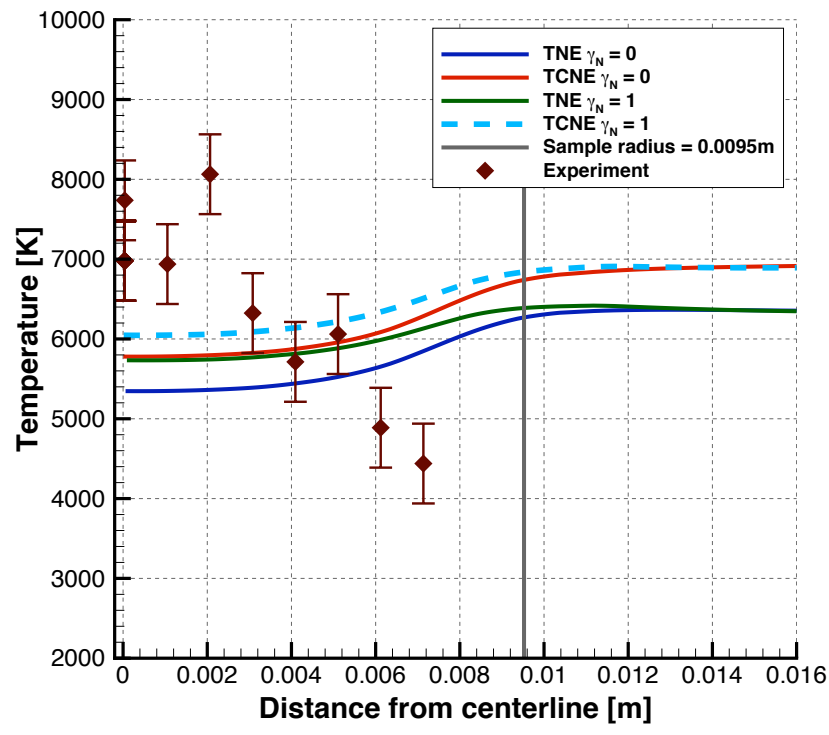

(a) Translational temperature



(b) Translational temperature contours

Figure 5. (a) Comparison of translational temperature between the computational and experimental data in the radial direction at a distance of $1.52 \mathrm{~mm}$ from the stagnation point. (b) Translational temperature contours for thermochemical nonequilibrium $(\gamma=1)$ showing the region of radial measurement. 


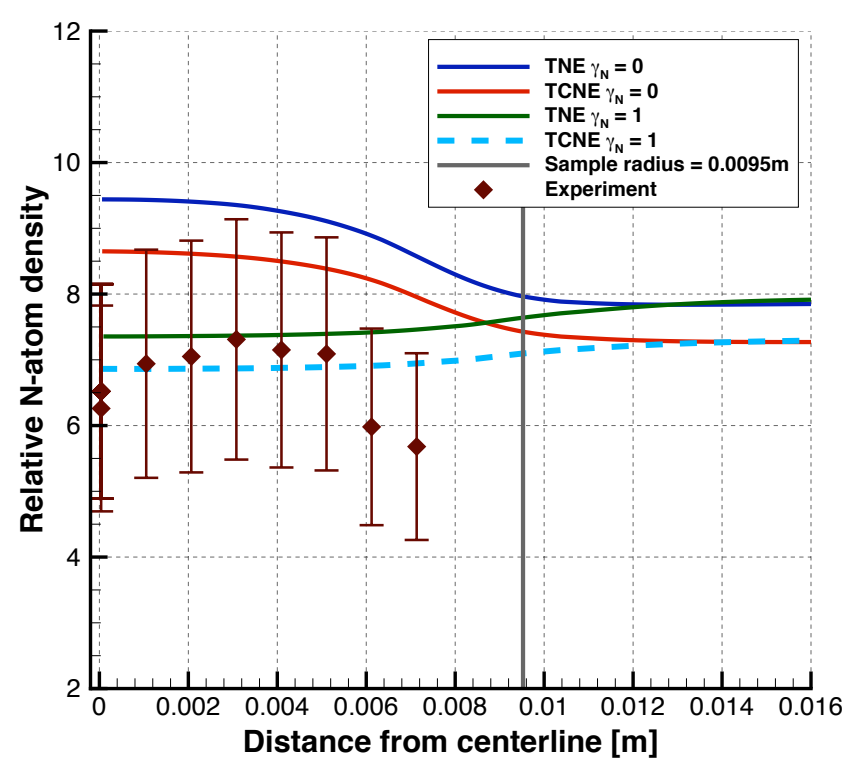

(a) Relative N-atom density

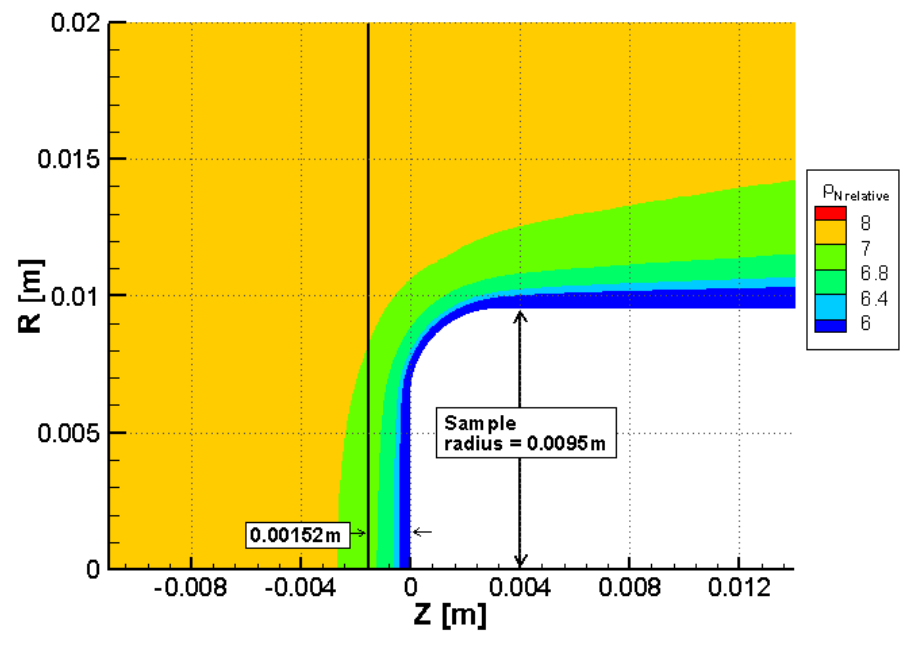

(b) Relative N-atom density contours

Figure 6. Comparison of relative N-atom density between the computational and experimental data in the radial direction at a distance of $1.52 \mathrm{~mm}$ from the stagnation point. (b) Relative N-atom density contours for thermochemical nonequilibrium $(\gamma=1)$ showing the region of radial measurement.

nitrogen atom density profiles for this case as shown in Figs. 4(b) and 6(a), respectively, show a loss in the number of nitrogen atoms in comparison with the thermal nonequilibrium case. The reason for this loss is explained by a small but finite net rate of recombination reactions in the chemically reacting nonequilibrium flow. Since the flow is chemically reacting, the dissociation-recombination reaction given by Eq. 6 is taken into account. Due to the high concentration of nitrogen atoms in the freestream, along with the relative long characteristic flow time associated with the subsonic velocity, recombination dominates over dissociation. This explanation is confirmed by plotting the normalized mixture total and species number densities for the thermal and thermochemical nonequilibrium cases as shown in Fig. 9(a). The number densities are normalized by their respective values at a distance of $4 \mathrm{~mm}$ (approximate thermal boundary layer thickness) from the stagnation point. The relative nitrogen atom, nitrogen molecule and total mixture number density overlay each other for thermal nonequilibrium. For the case of thermochemical nonequilibrium, the nitrogen atom number density increases slowly and the nitrogen molecule number density increases more rapidly than the total number density, indicating a net effect of recombination resulting in an increase in nitrogen molecule concentration. The influence of this chemical nonequilibrium can be seen in the increased wall heat flux in Fig. 7. As a result of recombination, some energy is transferred into the vibrational mode because of the addition of nitrogen molecules. Its effect can be seen in Fig. 7(c) by the increased vibrational convective heat flux for thermochemical nonequilibrium as compared to thermal nonequilibrium. The maximum contribution to the total heat flux is from the translational-rotational energy modes for these cases. The diffusive heat flux is negligible for all cases with a non-catalytic wall. Figure 8 shows a comparison of translational temperature contours between the thermochemical nonequilibrium (top half) and thermal nonequilibrium (bottom half) simulations. A rise in temperature can be seen along the stagnation line for the thermochemical nonequilibrium flow condition. This rise in temperature can be explained by the plot of nitrogen atom and molecule mole fractions along the stagnation line as shown in Fig. 9(b). An increase in nitrogen molecules and decrease in nitrogen atoms can be seen for thermochemical nonequilibrium and is explained by the effect of chemical nonequilibrium causing the release of energy due to recombination of nitrogen atoms to molecules and therefore causing a rise in temperature in the flow field.

\section{B. Effects of surface catalysis}

The effects of a fully-catalytic test article surface $(\gamma=1)$ on the boundary layer parameters and surface properties are discussed in this section. It can be seen in Fig. 4(b) that there is a decrease in relative 




(a) Total heat flux



(c) Vibrational convective heat flux



(b) Translational convective heat flux

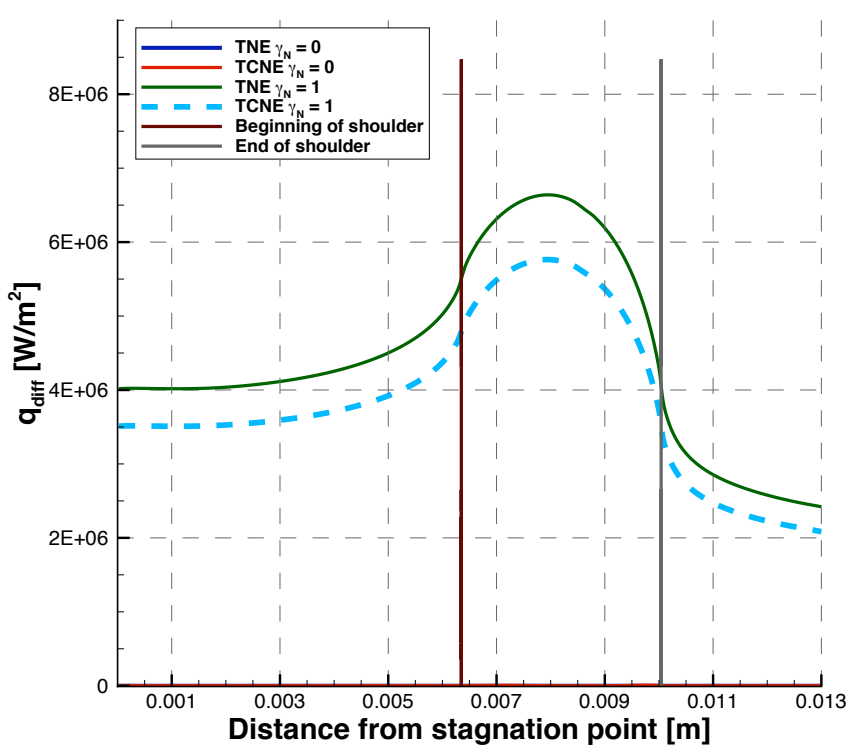

(d) Diffusive heat flux

Figure 7. Comparison of wall heat flux between the computational and experimental results for different test conditions listed in Table 3. 


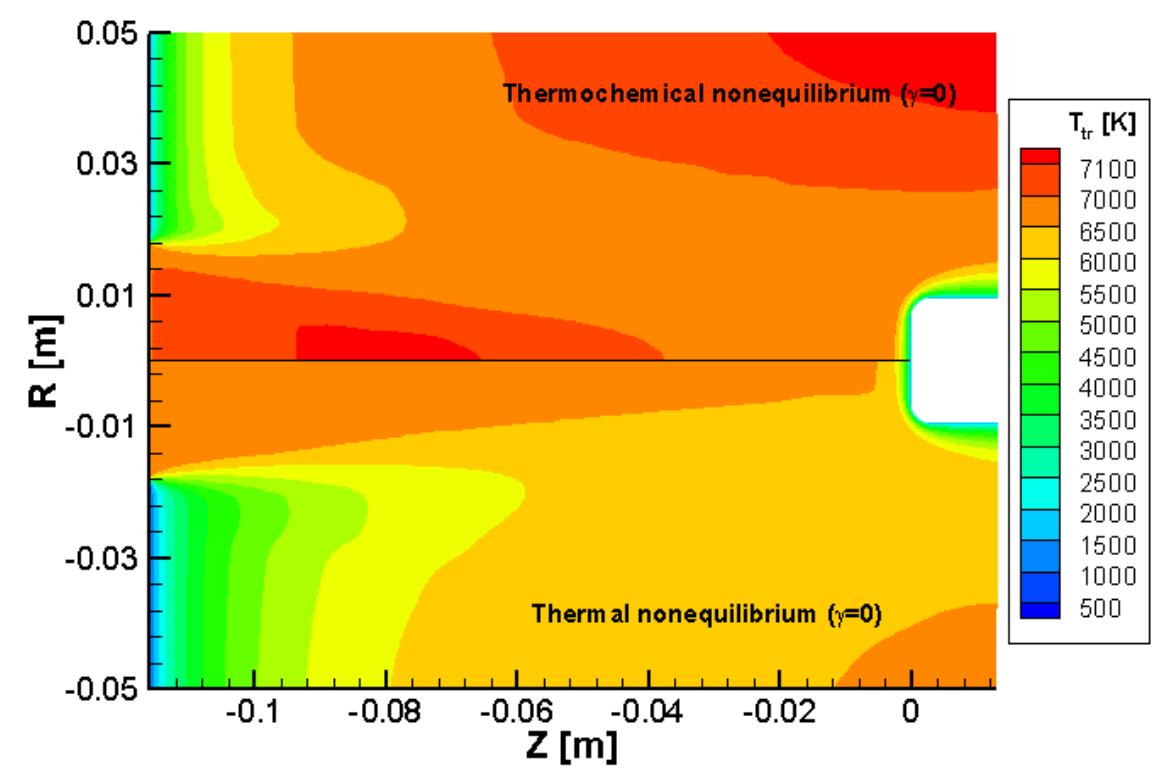

Figure 8. Comparison of translational temperature contours between thermochemical and thermal nonequilibrium simulation for a non-catalytic wall.

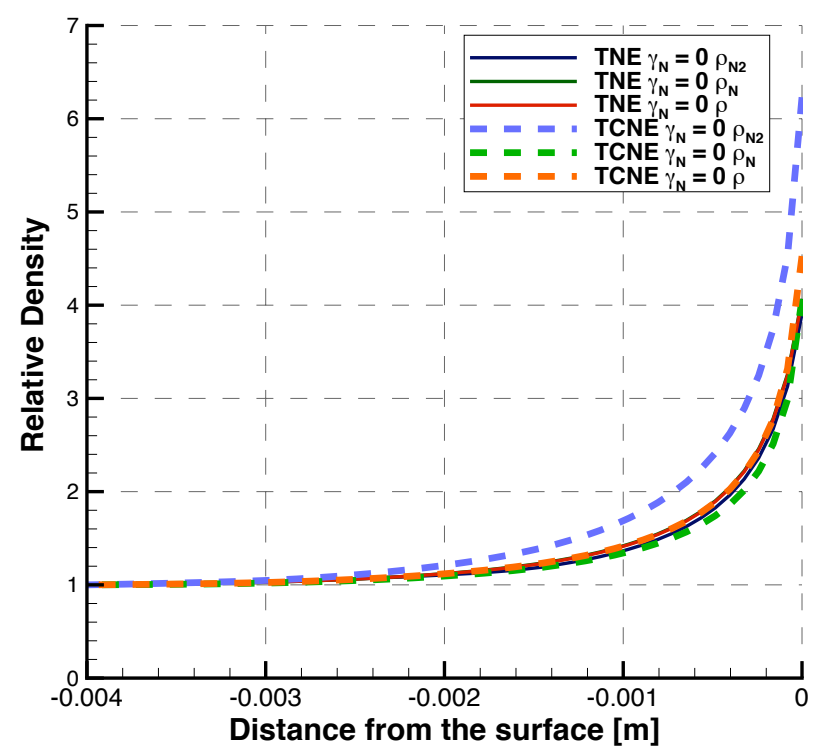

(a) Relative density

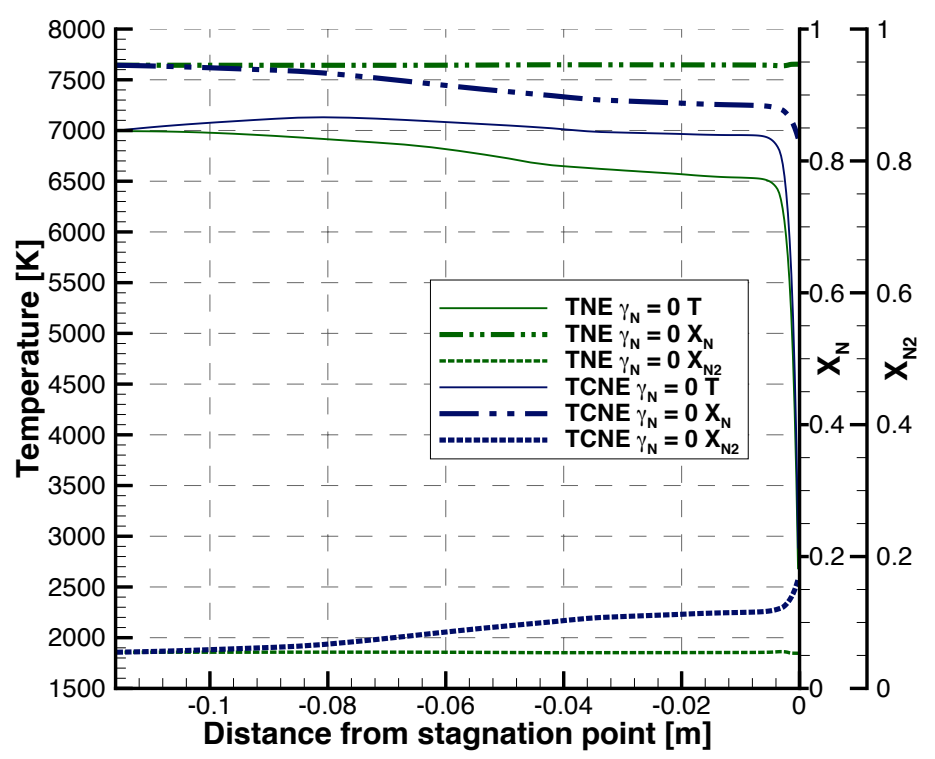

(b) Mole fraction

Figure 9. Computed stagnation streamline profiles obtained for a non-catalytic wall $\left(\gamma_{N}=0\right)$ : (a) relative mixture and species number density. (b) translational temperature and species mole fractions. 
nitrogen atom density in the experimental results which is at least partly explained by the presence of surface catalysis. To account for this, a numerical solution is obtained for thermal and thermochemical nonequilibrium with a fully-catalytic surface. The simulation run times for the thermal and thermochemical nonequilibrium cases are approximately 125 and 140 hours with each simulation using 48 processors. As seen in Figs. 4(a) and 5(a) for the case of thermochemical nonequilibrium, there is an $8 \%$ rise in temperature in the boundary layer in comparison with the thermal nonequilibrium non-catalytic wall. For this case, the stagnation line temperature profile has the closest agreement with the experimental data. The comparison of radial temperature profile between experimental and computational results (Fig. 5(a)) shows that the temperature decreases in the radial direction away from the stagnation line in the experimental results whereas the temperature increases in the computational results. The reason for this increase in the numerical results can be clearly explained from the temperature contours shown in Fig. 5(b) that includes the region where the radial measurement is taken. It can be seen that the temperature increases along the line at $1.52 \mathrm{~mm}$ from the stagnation point (indicated by the solid black line) and the reason for this is that the wall boundary effects decrease away from the sample. Similar decreasing wall boundary effects can be seen in the radial profiles of relative nitrogen atom density for all test cases and in the relative nitrogen atom density contours as shown in Figs. 6(a) and 6(b), respectively. The temperature and relative nitrogen atom density contours shown are for thermochemical nonequilibrium $(\gamma=1)$. Similar trends in the temperature and relative nitrogen atom density contours are observed for all the test cases. The different radial trends observed in the computational and experimental results may suggest the need to employ non-uniform inflow conditions for the ICP generated freestream.

The experimental temperature and relative nitrogen atom density values have uncertainties of about \pm 500 $\mathrm{K}$ and $\pm 25 \%$, respectively. ${ }^{25}$ A slight increase in temperature for thermal nonequilibrium $(\gamma=1)$ can be seen in Figs. 4(a) and 5(a), respectively. It can be seen that there is a decrease in the relative nitrogen atom density both for thermal and thermochemical nonequilibrium (Fig. 4(b) and 6(a)). For the thermal nonequilibrium case, the relative nitrogen atom density is slightly higher than for the thermochemical nonequilibrium flow as seen in Fig. 6(a). The reason for this is that the depletion of nitrogen atoms is caused by recombination to molecules as a result of a fully-catalytic wall in thermal nonequilibrium. For thermochemical nonequilibrium flow, in addition to recombination due to a fully-catalytic wall, the depletion of nitrogen atoms is caused by a recombination reaction due to chemical nonequilibrium as well. The computed relative nitrogen atom density is in close agreement with the measured experimental data both for the axial and radial profiles for a fully-catalytic wall. The total heat flux shown in Fig. 7 is maximum for thermal nonequilibrium followed by thermochemical nonequilibrium. The maximum contribution for both these cases is from the diffusive heat flux. The higher diffusive heat flux and therefore the total heat flux for thermal nonequilibrium can be explained by the higher number of nitrogen atoms reaching the surface and recombining as compared to the smaller number of nitrogen atoms reaching the surface because of loss due to recombination in thermochemical nonequilibrium. The addition of nitrogen molecules contributes to the vibrational convective heat flux and it can be seen that it is maximum for thermochemical nonequilibrium $(\gamma=1)$ as expected.

\section{Loss of nitrogen atoms}

The plausible causes of depletion of nitrogen atoms (Fig. 4(b) and 6(a)) observed in the measured experimental data and computational results for a fully catalytic wall are discussed in this section. The decreasing relative nitrogen atom density in the computational results for a fully-catalytic wall is explained by the strong presence of surface catalysis and recombination due to chemical nonequilibrium. However, in addition to these two processes, the loss of nitrogen atoms observed in the experiment may also be caused by a carbon nitridation reaction, where atomic nitrogen reacts with solid carbon and extracts it from the surface causing a loss in the mass of the sample. Sample mass loss is observed in the experiment and results for the surface recession are reported in the work by Lutz et al. ${ }^{23}$ This mechanism will be added to the computational model in future studies.

\section{Conclusion}

The purpose of this research was to study the catalytic gas-surface interactions for a graphite sample exposed to a subsonic high enthalpy nitrogen flow. Using the CFD code LeMANS, the effects of the flow field around the graphite sample and its surface properties were evaluated. Assessment of the computations 
was made using experimental tests that were conducted in the $30 \mathrm{~kW}$ Inductively Coupled Plasma facility at the University of Vermont. The first part of this study focussed on understanding the effects of thermal and thermochemical nonequilibrium on temperature, density and heat flux when the surface is considered non-catalytic. The results from simulations showed that thermal nonequilibrium effects were negligible and thermochemical nonequilibrium effects showed a rise in temperature, decrease in nitrogen atoms in the flow field, and an increase in the heat flux to the surface by a factor of 1.5. Therefore, it can be concluded that the flow studied is in a state of weak thermochemical nonequilibrium. The objective of the second part of the study was to determine the effects of surface catalysis. A simple binary atom recombination model was implemented to account for its effects. The use of this model showed that the heat flux transferred to the surface is increased approximately by a factor of 3.5 when the surface is fully-catalytic as opposed to a noncatalytic surface for thermochemical nonequilibrium. The final part of the study focussed on determining the causes for the decrease in nitrogen atom density in the boundary layer observed in the experimental results. The computational results obtained showed strong surface catalysis effects mostly caused by the depletion of nitrogen atoms in the boundary layer along with a weak recombination process as a result of chemical nonequilibrium.

This work is considered as the first step towards the development of a more accurate method to model the catalytic gas-surface interactions. A more accurate gas-surface interaction model should be added accounting for different elementary reactions that represent particle adsorption/desorption, the recombination of an atom of the gas with an atom adsorbed on the wall [Eley-Rideal (E-R) process] and recombination of two adsorbed atoms at the wall [Langmuir-Hinshelwood $(\mathrm{L}-\mathrm{H})$ process]. To validate numerical results with the ICP conditions, it is required to simulate flow in the test facility as closely as possible. First, in this work a zeroth order extrapolation is used to define the outflow boundary values. This condition is used for hypersonic flows for which the exit is always at supersonic speeds. The exit flow in the case of an ICP torch test facility is subsonic in nature. To study the effects of this outflow condition, a subsonic flow exit boundary condition should be implemented. A large flow domain is used for the presented work to account for this condition based on the suggestion from Prof. Phillip Roe. ${ }^{30}$ Second, a uniform velocity profile is used at the inlet, i.e. the exit of the ICP torch. A more accurate inlet velocity profile should be used to define the flow conditions more precisely. In this work, only the effect of convective heating is studied. It would be interesting to quantify the contribution of radiative heating.

\section{Acknowledgments}

The authors gratefully acknowledge funding for this work through Air Force Office of Scientific Research Grant FA-9550-11-1-0309. The use of supercomputers at the University of Michigan (NYX cluster) is essential to this work and is also greatly appreciated. The authors also acknowledge critical information provided for this research by Professor Doug Fletcher and his graduate students Andrew Lutz and Walt Owens at the University of Vermont.

\section{References}

\footnotetext{
${ }^{1}$ Marschall, J. and Fletcher, D. G., "High-Enthalpy Test Environments, Flow Modeling and In Situ Diagnostics for Characterizing Ultra-High Temperature Ceramics," Journal of the European Ceramic Society, Vol. 30, No. 11, 2010, pp. 23232336 .

${ }^{2}$ Laub, B. and Venkatapathy, E., "Thermal Protection System Technology and Facility Needs for Demanding Future Planetary Missions," International Workshop on Planetary Probe Atmospheric Entry and Descent Trajectory Analysis and Science, October 2003.

${ }^{3}$ Gnoffo, P. A., "Planetary-Entry Gas Dynamics," Vol. 31, 1999, pp. 459-94.

${ }^{4}$ Bose, D. and Wright, M., "Uncertainty Analysis of Laminar Aeroheating Predictions for Mars Entries," AIAA Paper 2005-4682, June 2005.

${ }^{5}$ Barbante, P., "Heat Flux Duplication Between Ground Facility and Hypersonic Flight," Journal of Thermophysics and Heat Transfer, Vol. 23, No. 4, October-December 2009, pp. 684-692.

${ }^{6}$ Alkandry, H. and Boyd, I. D., "Interactions of Single-Nozzle Sonic Propulsive Deceleration Jets on Mars Entry Aeroshells," Journal of Spacecraft and Rockets, Vol. 48, No. 4, 2011, pp. 564-572.

${ }^{7}$ Scalabrin, L. C. and Boyd, I. D., "Development of an Unstructured Navier-Stokes Solver for Hypersonic Nonequilibrium Aerothermodynamics," AIAA Paper 2005-5203, June 2005.

${ }^{8}$ Scalabrin, L. C. and Boyd, I. D., "Numerical Simulation of Weakly Ionized Hypersonic Flow for Reentry Configurations," AIAA Paper 2006-3773, June 2006.
} 
${ }^{9}$ Scalabrin, L. C. and Boyd, I. D., "Numerical Simulations of the FIRE-II Convective and Radiative Heating Rates," $A I A A$ Paper 2007-4044, June 2007.

${ }^{10}$ MacCormack, R. and Candler, G., "The Solution of the Navier-Stokes Equations Using Gauss-Siedel Line Relaxation," Computers and Fluids, Vol. 17, 1989, pp. 135-150.

${ }^{11}$ Karypis, G. and Kumar, V., "METIS: A Software Package for Partitioning Unstructured Graphs, Partitioning Meshes, and Computing Fill-Reducing Orderings of Sparse Matrices," University of Minnesota, 1998.

${ }^{12}$ T.D.Holman and I.D.Boyd, "Numerical Investigation of the Effects of Continuum Breakdown on Hypersonic Vehicle Surface Properties," AIAA Paper 2008-3928, June 2008.

${ }^{13}$ Scott, C. D., "Wall Catalytic Recombination and Boundary Conditions in Nonequilibrium Hypersonic Flow - With Applications," The Third Joint Europe/US Short Course in Hypersonic Flow, October 1990.

${ }^{14}$ Sarma, G., "Physio-chemical modelling in hypersonic flow simulation," Progress in Aerospace Sciences, Vol. 36, 2000, pp. 281-349.

${ }^{15}$ Godart, C., Salvetti, M. V., and Désidéri, J. A., "Computation of Catalycity Effects in Hypersonic Non-Equilibrium Flows," 2007.

${ }^{16}$ Bourdon, A. and Bultel, A., "Numerical Simulation of Stagnation Line Nonequilibrium Airflows for Reentry Applications," Journal of Thermophysics and Heat Transfer, Vol. 22, No. 2, April-June 2008, pp. 168-177.

${ }^{17}$ C.R.Wilke, "A Viscosity Equation for Gas Mixtures," Journal of Chemical Physics, Vol. 18, 1950, pp. 517-519.

${ }^{18}$ F.G.Blottner, Johnson, M., and Ellis, M., "Chemically Reacting Viscous Flow Program for Multi-Component Gas Mixtures," Sc-rr-70-754, Sandia Laboratories, Albuquerque, New Mexico, 1971.

${ }^{19}$ W.G.Vincenti and C.H.Kruger, Introduction to Physical Gas Dynamics, Krieger Publishing Company, 2002.

${ }^{20}$ P.A.Gnoffo, R.N.Gupta, and J.L.Shinn, "Conservation equations and physical models for hypersonic air flows in thermal and chemical nonequilibrium," Nasa-tp-2867, NASA Langley, Hampton, Virginia, 1989.

${ }^{21}$ C.Park, Nonequilibrium Hypersonic Aerothermodynamics, John Wiley \& Sons, 1990.

${ }^{22}$ K.Sutton and P.A.Gnoffo, "Multi Component Diffusion with application to computational aerothermodynamics," AIAA Paper 1998-2575, 1998.

${ }^{23}$ Lutz, A., Owens, W., Meyers, J., Fletcher, D., and Marschall, J., "Investigation of CN Production from Carbon Materials in Nitrogen Plasmas," AIAA Paper 2011-901, January 2011.

${ }^{24}$ Owens, W. P., Uhl, J., Dougherty, M., Lutz, A., Meyers, J., and Fletcher, D. G., "Development of a 30kW Inductively Coupled Plasma Torch for Aerospace Material Testing," AIAA Paper 2010-4322, June 2010.

${ }^{25}$ Fletcher, D., personal communication, Mechanical Engineering, University of Vermont, Burlington,VT.

${ }^{26} \mathrm{McBride}, \quad$ B. J. and Gordon, S., "Chemical Equilibrium with Applications (CEA)," [http://www.grc.nasa.gov/WWW/CEAWeb/], accessed in 2011.

${ }^{27}$ Singh, H., Coburn, J. W., and Graves, D. B., "Recombination coefficients of O and N radicals on stainless steel," Journal of Applied Physics, Vol. 88, No. 3748, 2000.

${ }^{28}$ Adams, S. F. and Miller, T. A., "Surface and volume loss of atomic nitrogen in a parallel plate rf discharge reactor," Plasma Sources Sci. Technol., Vol. 9, 2000, pp. 1-8.

${ }^{29}$ Pointwise Version 16.04, Pointwise 2011.

${ }^{30}$ Roe, P., personal communication, Aerospace Engineering, University of Michigan, Ann Arbor,MI. 